\title{
High production of 2,3-butanediol from biodiesel-derived crude glycerol by metabolically engineered Klebsiella oxytoca M1
}

\author{
Sukhyeong Cho ${ }^{1}$, Taeyeon Kim, ${ }^{1,}$, Han Min Woo ${ }^{1,3}$, Yunje Kim ${ }^{1}$, Jinwon Lee ${ }^{4}$ and Youngsoon Um ${ }^{1,3^{*}}$
}

\begin{abstract}
Background: 2,3-Butanediol (2,3-BDO) is a promising bio-based chemical because of its wide industrial applications. Previous studies on microbial production of 2,3-BDO has focused on sugar fermentation. Alternatively, biodieselderived crude glycerol can be used as a cheap resource for 2,3-BDO production; however, a considerable formation of 1,3-propanediol (1,3-PDO) and low concentration, productivity, and yield of 2,3-BDO from glycerol fermentation are limitations.

Results: Here, we report a high production of 2,3-BDO from crude glycerol using the engineered Klebsiella oxytoca $\mathrm{M} 3$ in which pduC (encoding glycerol dehydratase large subunit) and IdhA (encoding lactate dehydrogenase) were deleted to reduce the formation of 1,3-PDO and lactic acid. In fed-batch fermentation with the parent strain K. oxytoca $\mathrm{M1}$, crude glycerol was more effective than pure glycerol as a carbon source in 2,3-BDO production (59.4 vs. $73.8 \mathrm{~g} / \mathrm{L}$ ) and by-product reduction (1,3-PDO, 8.9 vs. $3.7 \mathrm{~g} / \mathrm{L}$; lactic acid, $18.6 \mathrm{vs.} 9.8 \mathrm{~g} / \mathrm{L})$. When the double mutant was used in fed-batch fermentation with pure glycerol, cell growth and glycerol consumption were significantly enhanced and 2,3-BDO production was 1.9-fold higher than that of the parent strain (59.4 vs. $115.0 \mathrm{~g} / \mathrm{L}$ ) with $6.9 \mathrm{~g} / \mathrm{L} \mathrm{of} \mathrm{1,3-PDO} \mathrm{and} \mathrm{a}$ small amount of lactic acid ( $0.7 \mathrm{~g} / \mathrm{L})$. Notably, when crude glycerol was supplied, the double mutant showed 1,3-PDOfree 2,3-BDO production with high concentration $(131.5 \mathrm{~g} / \mathrm{L})$, productivity $(0.84 \mathrm{~g} / \mathrm{L} / \mathrm{h})$, and yield $(0.44 \mathrm{~g} / \mathrm{g}$ crude glycerol). This result is the highest 2,3-BDO production from glycerol fermentation to date.
\end{abstract}

Conclusions: $2,3-\mathrm{BDO}$ production from glycerol was dramatically enhanced by disruption of the $p d u C$ and IdhA genes in $\mathrm{K}$. oxytoca M1 and 1,3-PDO-free 2,3-BDO production was achieved by using the double mutant and crude glycerol. 2,3-BDO production obtained in this study is comparable to 2,3-BDO production from sugar fermentation, demonstrating the feasibility of economic industrial 2,3-BDO production using crude glycerol.

Keywords: 2,3-butanediol, Klebsiella oxytoca, Biodiesel-derived crude glycerol, Fermentation

\section{Background}

In the last few years, considerable effort and progress have been made in the production of bio-based bulk chemicals from renewable resources because of the decrease in fossil fuel availability and increasing concern for global warming [1]. 2,3-Butanediol (2,3-BDO)

\footnotetext{
*Correspondence: yum@kist.re.kr

${ }^{3}$ Clean Energy and Chemical Engineering, Korea University of Science and Technology, 217 Gajeong-ro, Yuseong-gu, Daejeon, Republic of Korea Full list of author information is available at the end of the article
}

is a promising bio-based bulk chemical due to numerous industrial applications, such as the manufacture of printing inks, perfumes, softening and moistening agents, pharmaceuticals, anti-freeze agent, and liquid fuels [2, 3]. In addition, methyl ethyl ketone (an organic solvent for resins and lacquers) and 1,3-butadiene (a monomer for synthetic rubber) can be produced by the hydration of 2,3-BDO [3, 4].

Previous studies on biological production of 2,3-BDO have focused on sugar fermentation using glucose [5-10] 
and sucrose [11] as the carbon sources. Because of the relatively high cost of conventional sugars, 2,3-BDO production has been investigated using a non-edible inexpensive lignocellulosic biomass and organic waste, such as corncobs [12], corn stover [13], Jerusalem artichoke tubers [14], Jatropha hulls [15], and sugarcane molasses [16].

Glycerol, which is generated as a by-product from ethanol fermentation, fat saponification, and biodiesel production [17], is also an attractive cheap resource for 2,3-BDO production. In particular, because the amount of biodiesel-derived crude glycerol is almost equivalent to $10 \%(\mathrm{w} / \mathrm{w})$ of global biodiesel production, there is an increasing surplus of glycerol on the world market [18]. Klebsiella species, such as K. pneumoniae and $K$. oxytoca, have been found to utilize glycerol as the sole carbon source and produce 1,3-propanediol (1,3-PDO) and 2,3-BDO [18-22]. Conversion of glycerol to 2,3-BDO occurs through the oxidative pathway, where glycerol is converted to dihydroxyacetone phosphate (DHAP) via glycerol-3-phosphate in the presence of electron acceptors (e.g., $\mathrm{O}_{2}$ under aerobic conditions) or via dihydroxyacetone in the absence of oxygen (i.e., fermentative route) (Fig. 1) [23]. In addition to the oxidative branch, glycerol is also metabolized through the reductive pathway, which results in the generation of 1,3-PDO (Fig. 1). 1,3-PDO is a major by-product generated during the production of 2,3-BDO using glycerol and may serve as an obstacle for obtaining a high purity of 2,3-BDO in downstream processes because of the similar boiling points of 2,3-BDO and 1,3-PDO [3].

Several reports have investigated the effect of dissolved oxygen level [24] and $\mathrm{pH}[20,21]$ on 2,3-BDO production and the ratio of 1,3-PDO:2,3-BDO using glycerol as the sole carbon source. Until now, Petrov and Petrova [21] have reported the highest 2,3-BDO production $(70 \mathrm{~g} / \mathrm{L})$ from pure glycerol by $\mathrm{K}$. pneumoniae at the productivity of $0.47 \mathrm{~g} / \mathrm{L} / \mathrm{h}$ through forced $\mathrm{pH}$ fluctuation (consecutive raising of $\mathrm{pH}$ with $\Delta \mathrm{pH}=1$ at every $12 \mathrm{~h}$ using $5 \mathrm{~N}$ sodium hydroxide); however, 1,3-PDO was still generated up to $16.3 \mathrm{~g} / \mathrm{L}$, and this result was obtained using pure glycerol, not crude glycero1. Yang et al. [25] demonstrated 1,3-PDO-free 2,3-BDO production (83.3 and $0.87 \mathrm{~g} / \mathrm{L} / \mathrm{h}$ ) using Bacillus amyloliquefaciens by supplying beet molasses as a co-substrate in addition to biodieselderived crude glycerol; however, when biodiesel-derived glycerol was supplied as the sole carbon source, much lower 2,3-BDO concentration $(43.1 \mathrm{~g} / \mathrm{L})$ and productivity $(0.45 \mathrm{~g} / \mathrm{L} / \mathrm{h})$ were obtained by $B$. amyloliquefaciens. Moreover, considering that the 2,3-BDO titer and productivity levels obtained from glucose fermentation by $K$. pneumoniae (101.5-150.0 g/L and 2.54-4.21 g/L/h) [5, 7] and $K$. oxytoca $(113-130 \mathrm{~g} / \mathrm{L}$ and $1.63-2.1 \mathrm{~g} / \mathrm{L} / \mathrm{h})[6,8]$ were much higher than those obtained using glycerol as the sole carbon source, much improvement in 2,3-BDO production from crude glycerol is required to facilitate a cost-effective high 2,3-BDO production.

Previously, a newly isolated $K$. oxytoca M1 was reported to be able to produce 2,3-BDO and acetoin selectively as the main products depending on temperature [26]. In this study, we attempted a high 2,3-BDO production using biodiesel-derived crude glycerol as the sole carbon source by the metabolically engineered $K$. oxytoca M3, in which $p d u C$ (encoding glycerol dehydratase large subunit, accession number AIE72369) and $l d h A$ (encoding lactate dehydrogenase, accession number AIE70186) were deleted to reduce the formation of by-products, such as 1,3-PDO and lactic acid. The double deletion mutant showed a significantly improved 2,3-BDO production from pure glycerol and crude glycerol in fed-batch fermentation compared to the parent strain. Notably, 1,3-PDO-free 2,3-BDO production by the double deletion mutant was observed when crude glycerol was used as the carbon source in fed-batch fermentation. To our knowledge, the final titer $(131.5 \mathrm{~g} / \mathrm{L})$, the productivity $(0.84 \mathrm{~g} / \mathrm{L} / \mathrm{h})$, and the yield ( $0.44 \mathrm{~g} / \mathrm{g}$ crude glycerol) of 2,3-BDO from crude glycerol obtained by the double deletion mutant were the highest recorded in 2,3-BDO production from glycerol as the sole carbon source to date.

\section{Results and discussion}

\section{Flask fermentation of $K$. oxytoca M1 using pure and crude} glycerol

To evaluate glycerol utilization and 2,3-BDO production by $K$. oxytoca M1, flask batch fermentation was performed with pure glycerol and crude glycerol as the sole carbon sources at the initial concentration of $35-40 \mathrm{~g} / \mathrm{L}$. As shown in Table 1, the performance of the 2,3-BDO production by $K$. oxytoca M1 was similar regardless of the type of glycerol (Table 1). Cell growth (dry cell weight, DCW) appeared to be inhibited by crude glycerol. Several components in crude glycerol, such as free fatty acid, methanol, MONG (matter organic non-glycerol), and salt, are known to cause cell growth inhibition $[27,28]$. However, considering that the final $\mathrm{pH}$ of crude glycerol fermentation was lower than the $\mathrm{pH}$ value of pure glycerol fermentation ( $\mathrm{pH} 4.9 \mathrm{vs} \mathrm{pH} 6.0$ ), a lower DCW in crude glycerol fermentation might be caused by a low $\mathrm{pH}$ level as presented in the next section. Overall, K. oxytoca M1 could produce 2,3-BDO as the main product using glycerol. Notably, 1,3-PDO, which is known to be one of the main by-products in 2,3-BDO fermentation by Klebsiella species [19-21], was not detected for $K$. oxytoca M1 in flask fermentation. Therefore, $K$. oxytoca M1 was further investigated as a potential strain for 2,3$\mathrm{BDO}$ production from glycerol. 


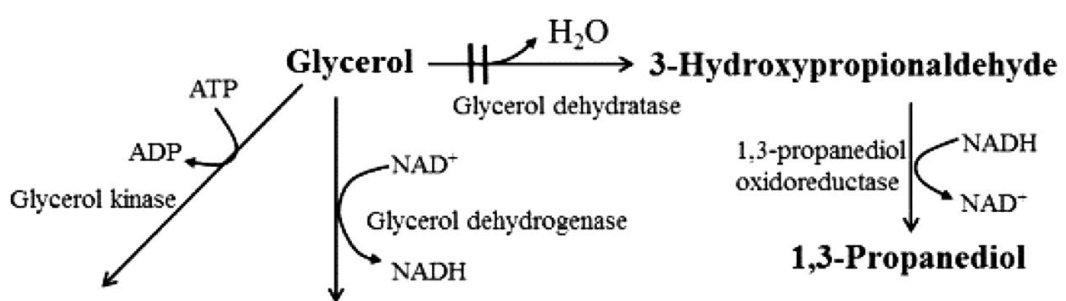

\section{Glycerol-3-phosphate Dihydroxyacetone}
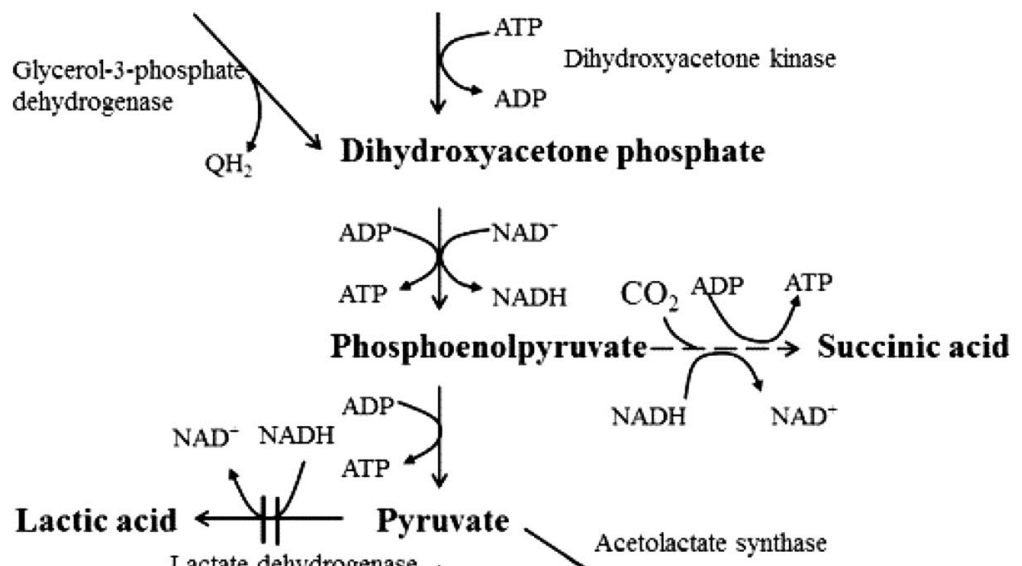

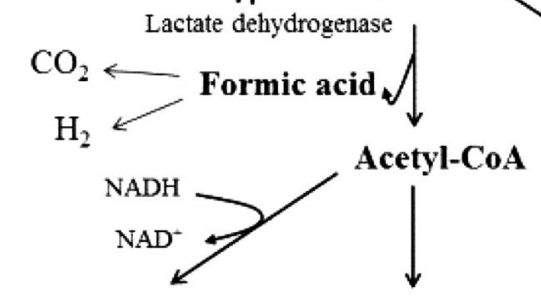

Acetaldehyde

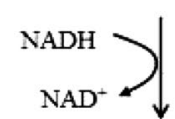

Ethanol
Acetyl-(D)

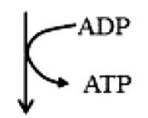

Acetic acid

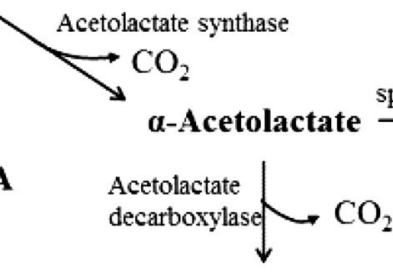

R-acetoin

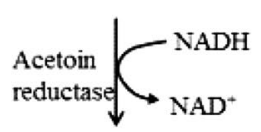

Meso-2,3-butanediol

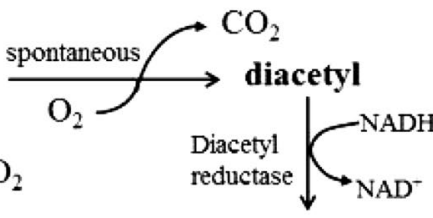

S-acetoin

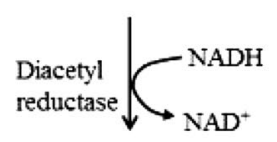

(2S,3S)-2,3-butanediol

Fig. 1 Metabolic pathway of 2,3-BDO from glycerol in K. oxytoca M1. The crossed double line is the pathway deleted in this study

Table 1 Comparison of flask batch fermentations by $K$. oxytoca M1 using pure and crude glycerol

\begin{tabular}{lll}
\hline & Pure glycerol & Crude glycerol \\
\hline Glycerol consumption $(\mathrm{g} / \mathrm{L})$ & $33.9 \pm 0.27$ & $37.8 \pm 0.74$ \\
Dry cell weight $(\mathrm{g} / \mathrm{L})$ & $2.2 \pm 0.29$ & $1.6 \pm 0.10$ \\
Lactic acid $(\mathrm{g} / \mathrm{L})$ & $0.3 \pm 0.59$ & $4.2 \pm 0.13$ \\
Ethanol $(\mathrm{g} / \mathrm{L})$ & $2.5 \pm 0.39$ & $3.1 \pm 0.13$ \\
1,3-PDO (g/L) & 0 & 0 \\
2,3-BDO (g/L) & $8.4 \pm 0.46$ & $8.9 \pm 0.26$ \\
2,3-BDO productivity (g/L/H) & $0.17 \pm 0.01$ & $0.19 \pm 0.01$ \\
2,3-BDO yield (g/g) & $0.25 \pm 0.02$ & $0.24 \pm 0.00$ \\
Final pH & $6.0 \pm 0.04$ & $4.9 \pm 0.01$ \\
Time (h) & 48 & 48 \\
\hline
\end{tabular}

The data are given as average \pm standard deviation of triplicate experiments
Fed-batch fermentations of $K$. oxytoca M1 using pure and crude glycerol

To investigate the feasibility of a high 2,3-BDO production by K. oxytoca M1 from glycerol, fed-batch fermentation using pure glycerol was conducted with $\mathrm{pH}$ control $(\mathrm{pH}=6)$ in a 3 L-bioreactor. Fed-batch fermentation using crude glycerol was also performed to investigate whether the accumulation of impurities in crude glycerol would inhibit the cell growth and 2,3-BDO production of $K$. oxytoca M1. When fed-batch fermentation using pure glycerol was carried out for $114 \mathrm{~h}$, K. oxytoca M1 successfully produced 2,3-BDO at concentrations up to $59.4 \mathrm{~g} / \mathrm{L}$ with the productivity of $0.52 \mathrm{~g} / \mathrm{L} / \mathrm{h}$ (Table 2; Fig. 2a). Interestingly, when crude glycerol was used in fermentation, DCW (6.1 g/L) and 2,3-BDO concentrations 
Table 2 Comparison of fed-batch fermentations by $K$. oxytoca M1 and K. oxytoca M3 using pure and crude glycerol

\begin{tabular}{|c|c|c|c|c|}
\hline \multirow[t]{2}{*}{ Strain } & \multicolumn{2}{|l|}{ K. oxytoca M1 } & \multicolumn{2}{|l|}{ K. oxytoca M3 } \\
\hline & Pure glycerol & Crude glycerol & Pure glycerol & Crude glycerol \\
\hline Glycerol consumption (g/L) & 189.5 & 177.7 & 297.1 & 300.3 \\
\hline Dry cell weight (g/L) & 4.1 & 6.1 & 5.8 & 9.0 \\
\hline 2,3-BDO (g/L) & 59.4 & 73.8 & 115.0 & 131.5 \\
\hline 1,3-PDO (g/L) & 8.9 & 3.7 & 6.9 & 0 \\
\hline Lactic acid (g/L) & 18.6 & 9.8 & 0.7 & 0.8 \\
\hline Ethanol $(\mathrm{g} / \mathrm{L})$ & 4.0 & 1.9 & 9.3 & 1.7 \\
\hline 2,3-BDO productivity (g/L/h) & 0.52 & 0.68 & 1.01 & 0.84 \\
\hline 2,3-BDO yield $(\mathrm{g} / \mathrm{g})$ & 0.31 & 0.42 & 0.39 & 0.44 \\
\hline Fermentation time (h) & 114 & 109 & 114 & 156 \\
\hline
\end{tabular}

(73.8 $\mathrm{g} / \mathrm{L})$ as well as $2,3-\mathrm{BDO}$ productivity $(0.68 \mathrm{~g} / \mathrm{L} / \mathrm{h})$ were much higher than those achieved using pure glycerol (Table 2; Fig. 2b). Similarly, it has been reported that crude glycerol exhibited positive effects on glycerol consumption and 1,3-PDO production for $K$. pneumoniae [27]. Until now, $70 \mathrm{~g} / \mathrm{L}$ has been the highest reported 2,3BDO concentration from pure glycerol as the sole carbon source at a productivity of $0.47 \mathrm{~g} / \mathrm{L} / \mathrm{h}$ using forced $\mathrm{pH}$ fluctuations $(\Delta \mathrm{pH}=1$ at every $12 \mathrm{~h})$ [21]. In this study, higher concentration $(73.8 \mathrm{~g} / \mathrm{L})$ and productivity $(0.68 \mathrm{~g} / \mathrm{L} / \mathrm{h})$ in comparison to previous results were obtained by simply maintaining the $\mathrm{pH}$ level at 6 and using crude glycerol.

Lactic acid and 1,3-PDO productions during the fedbatch fermentation (Table 2) were different from those observed during flask fermentation (Table 1). Lactic acid production from pure glycerol rapidly increased to $30.5 \mathrm{~g} / \mathrm{L}$ after $40 \mathrm{~h}$ and decreased to $18.6 \mathrm{~g} / \mathrm{L}$ (Fig. 2a). The production of lactic acid from crude glycerol was higher than that with pure glycerol during $24 \mathrm{~h}$ of fermentation (35.7 $\mathrm{g} / \mathrm{L}$ at $24 \mathrm{~h}$ ), but decreased to $9.8 \mathrm{~g} / \mathrm{L}$ at $109 \mathrm{~h}$. In contrast to the flask fermentation results (Table 1), 1,3PDO was produced in fed-batch fermentation from both pure and crude glycerol with concentrations up to 8.9 and $3.7 \mathrm{~g} / \mathrm{L}$, respectively (Table 2 ). Co-production of 1,3PDO and lactic acid along with 2,3-BDO production has been generally observed in the fermentation of Klebsiella species using glycerol as the sole carbon source [20, 21, 29]. Previous studies have reported that the product distribution greatly depended on the $\mathrm{pH}$ level; 1,3-PDO and lactic acid formation tended to increase with $\mathrm{pH}$ control at 7 , while 2,3-BDO production increased without $\mathrm{pH}$ control (final pH $=4.3-5.1$ ) [20]. In the fed-batch fermentation of $K$. oxytoca M1, because the $\mathrm{pH}$ level was kept at 6 , it might have caused the increase of 1,3-PDO and lactic acid formation in comparison with flask cultivation. When fed-batch fermentation of $K$. oxytoca M1 was conducted without $\mathrm{pH}$ control using pure glycerol,
2,3-BDO was produced mainly with a trace of 1,3-PDO and lactic acid; but, once $\mathrm{pH}$ was decreased below 4.9, glycerol was not utilized anymore at that point (data not shown). Thus, further fed-batch fermentation was conducted with $\mathrm{pH}$ control at 6 .

\section{Construction of the pduC deletion mutant and batch fermentation of the mutant using pure glycerol}

Although $K$. oxytoca M1 could produce 2,3-BDO using crude glycerol at a higher concentration and productivity levels than those achieved in previous studies, by-product formation needed to be decreased for efficient conversion of glycerol to 2,3-BDO. To reduce the formation of 1,3-PDO and lactic acid, the genes encoding glycerol dehydratase large subunit $(\mathrm{PduC})$, which is responsible for the first step of 1,3-PDO synthesis from glycerol, and lactate dehydrogenase (LDH) converting pyruvate to lactic acid were chosen for deletion.

Even though blocking 1,3-PDO formation seems necessary for 2,3-BDO production from glycerol, there has been no study regarding the effect of the deletion of $p d u C$, the gene encoding PduC, on cell growth and 2,3BDO production. Thus, to investigate whether the deletion of $p d u C$ exhibited adverse effects on cell growth and 2,3-BDO production, $K$. oxytoca M1 pduC deletion mutant was first constructed using the $\lambda$ Red recombination system [30]. PCR result and nucleotide sequencing data confirmed that the $p d u C$ gene of K. oxytoca M1 was successfully deleted (Figs. 1a) and this mutant strain was named $K$. oxytoca M2 (Table 3).

When batch fermentation was conducted with $K$. oxytoca M2 strain with the $\mathrm{pH}$ level maintained at 6.0 (Fig. 3), $p d u C$ deletion showed a positive effect on cell growth and 2,3-BDO production compared to the parent strain. Moreover, the deletion of $p d u C$ gene of $K$. oxytoca M1 resulted in nearly abolished 1,3-PDO formation $(0.8 \mathrm{~g} / \mathrm{L}$ of $1,3-\mathrm{PDO})$ in comparison with the 1,3 PDO production of the parent strain $(7.2 \mathrm{~g} / \mathrm{L}$ at $36 \mathrm{~h}$ in 

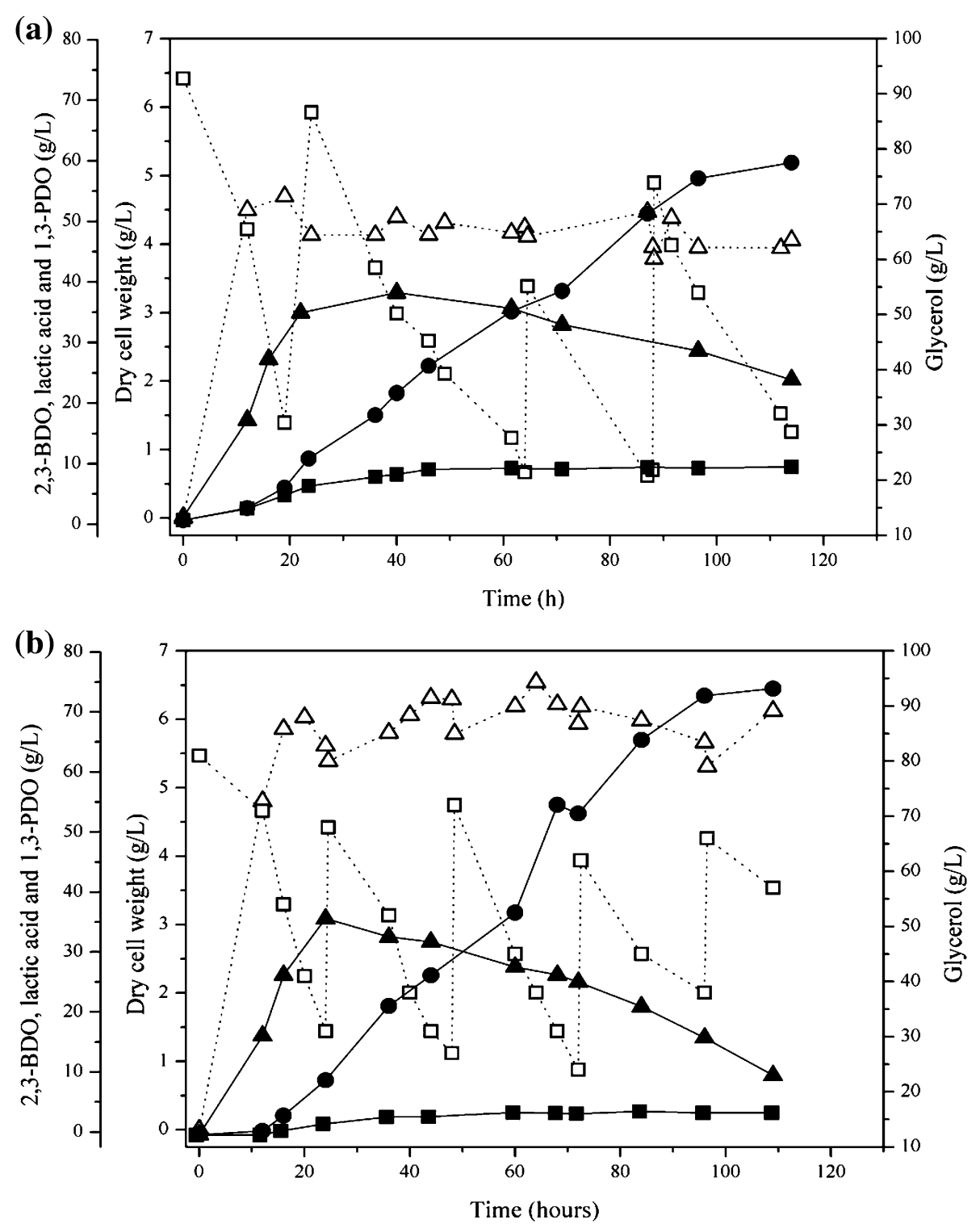

Fig. 2 Time course of 2,3-BDO fermentation using pure glycerol and crude glycerol by K. oxytoca M1. a Fed-batch fermentation with pure glycerol. b Fed-batch fermentation with crude glycerol. The following symbols were used: dry cell weight (unfilled triangle), residual glycerol (unfilled square), 2,3-BDO (filled circle), 1,3-PDO (filled square), and lactic acid (filled triangle)

Fig. 2a). This demonstrates that the disruption of $p d u C$ was effective for the reduction of 1,3-PDO formation. However, lactic acid was still produced in concentrations up to $30 \mathrm{~g} / \mathrm{L}$ at $21.5 \mathrm{~h}$, and 2,3-BDO production was not significantly improved in comparison with the parent strain.

\section{Construction of $p d u C$ and $I d h A$ double deletion mutant and fed-batch fermentation of the double mutant using pure glycerol}

To reduce lactic acid production, we generated the double deletion mutant strain $K$. oxytoca M1 $\Delta p d u C \Delta l d h A$ (i.e., K. oxytoca M3) in which the $l d h A$ gene was abrogated from the $p d u C$ mutant (i.e., $K$. oxytoca M2) (Table 3). Successful deletion of $l d h A$ was confirmed by PCR amplification of the $l d h A$ flank region with the primers ldhAcon1 and ldhAcon2 (steps 2 and 3 in Additional file 1: Fig. S1B) and nucleotide sequencing data. While the fragment of $1100 \mathrm{bp}$ (Additional file 1: Fig. S2, lane 4) containing the intact $l d h A$ gene was amplified from the parent strain, the fragment of $150 \mathrm{bp}$ (Additional file 1: Fig. S2, lane 5) was identified in the mutant strain using the primers ldhAcon 1 and ldhAcon2. This mutant strain was named $K$. oxytoca M3 (Table 3). 
Table 3 Bacterial strains and plasmids used in this study

\begin{tabular}{|c|c|c|}
\hline Strains or plasmids & Genotype and relevant characteristics & Source or references \\
\hline \multicolumn{3}{|l|}{ Strains } \\
\hline K. oxytoca M1 & Parent strain & {$[26]$} \\
\hline K. oxytoca M2 & K. oxytoca M1 $\Delta$ pduC & This study \\
\hline K. oxytoca M3 & K. oxytoca M1 $\Delta p d u C \Delta / d h A$ & This study \\
\hline K. oxytoca KCTC1686 & Type strain & [33] \\
\hline E. coli $\mathrm{DH} 5 \alpha$ & supE44, $\Delta$ lacU169, ( $\varphi 80$ lacZ $\Delta \mathrm{M} 15)$, hsdR17, recA1, endA1, gyrA96, thi-1, relA1 & Invitrogen \\
\hline \multicolumn{3}{|l|}{ Plasmids } \\
\hline pRedET & Derivative of pSC101, Tet $^{R}$, temperature sensitive, carrying lambda red recombinase & Gene bridge \\
\hline 707-FLPe & Derivative of pSC101, Tet $^{R}$, temperature sensitive, containing an FLPe recombinase & Gene bridge \\
\hline PTOP-FCF & Derivative of pUC, containing an FRT-flanked $\mathrm{Cm}^{\mathrm{R}}$ cassette-involved vector & {$[34]$} \\
\hline
\end{tabular}

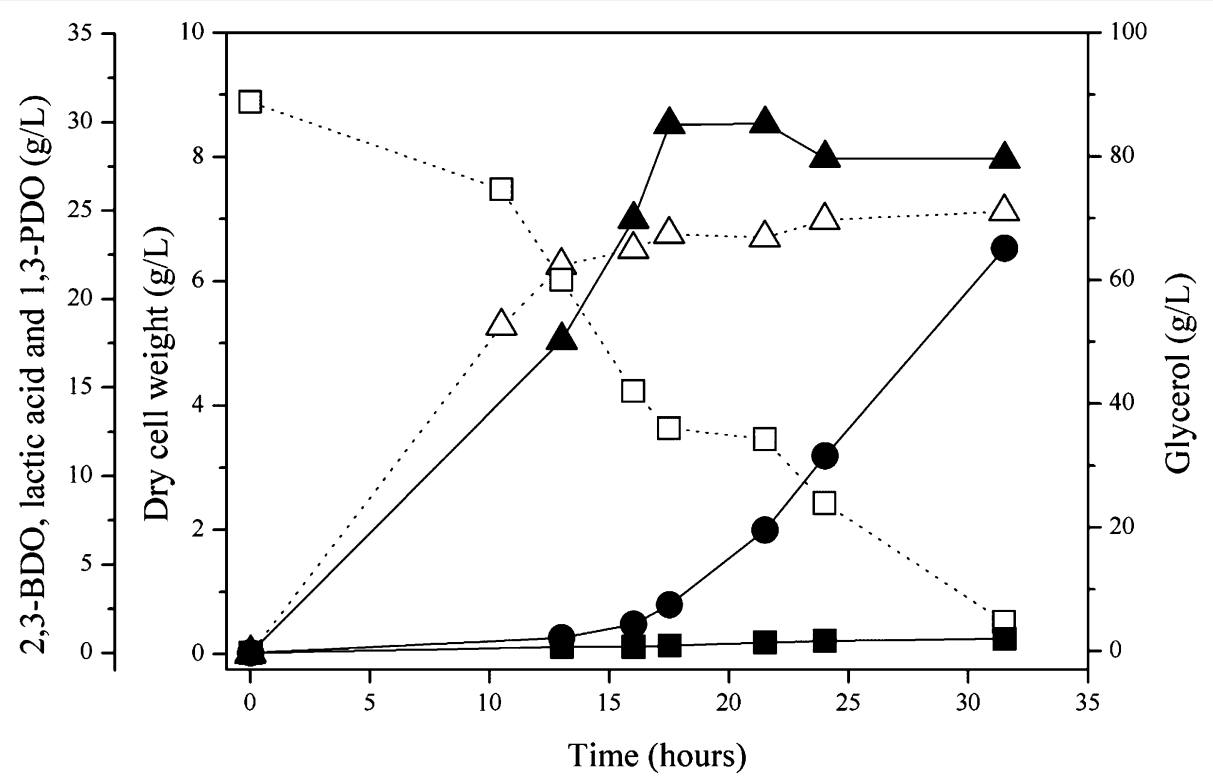

Fig. 3 Time course of 2,3-BDO fermentation using pure glycerol by K. oxytoca M1 $\Delta$ pduC. The following symbols were used: dry cell weight (unfilled triangle), residual glycerol (unfilled square), 2,3-BDO (filled circle), 1,3-PDO (filled square), and lactic acid (filled triangle)

To investigate the effect of the deletion of $p d u C$ and $l d h A$ on 2,3-BDO production, fed-batch fermentation was conducted with pure glycerol using $K$. oxytoca M3 and the results were compared with the fed-batch fermentation results of the parent strain (K. oxytoca M1, Fig. 2a). The fed-batch fermentation was operated over $135 \mathrm{~h}$, but glycerol consumption and 2,3-BDO production were negligible after $114 \mathrm{~h}$. As shown in Table 2, the total amount of glycerol consumed increased in comparison with that of the parent strain (297.1 g/L by K. oxytoca M3 vs. $189.5 \mathrm{~g} / \mathrm{L}$ by $K$. oxytoca M1). The maximum DCW of $K$. oxytoca M3 was also greater than that of $K$. oxytoca M1 $(8.6 \mathrm{~g} / \mathrm{L}$ vs. $4.7 \mathrm{~g} / \mathrm{L}$ ) (Figs. 2a, 4a). These results clearly indicate that the deletion of $p d u C$ and $l d h A$ positively affected glycerol uptake and cell growth. More importantly, the disruption of $l d h A$ resulted in a nearly abolished lactic acid production $(0.7 \mathrm{~g} / \mathrm{L}$, Table 2$)$, and $2,3-\mathrm{BDO}$ production was remarkably increased up to 1.9 -fold of the parent strain (59.4 vs. $115.0 \mathrm{~g} / \mathrm{L}$ ). The yield of 2,3-BDO with $K$. oxytoca M3 was also much higher than that of $K$. oxytoca M1 owing to the remarkable reduction of lactic acid production (Table 2). Carbon recovery to 2,3-BDO was $53 \%$ (mole/mole) of the consumed glycerol and the rest of the carbon would be incorporated to $\mathrm{CO}_{2}\left(2 \mathrm{~mol}\right.$ of $\mathrm{CO}_{2}$ emission per mole 2,3-BDO production), cell mass, and by-products (1,3-PDO, lactic acid, ethanol, etc.).

Regarding the formation of by-products, the concentration of ethanol was increased ( $4.0 \mathrm{vs.} 9.3 \mathrm{~g} / \mathrm{L}$ ) (Table 2$)$ 

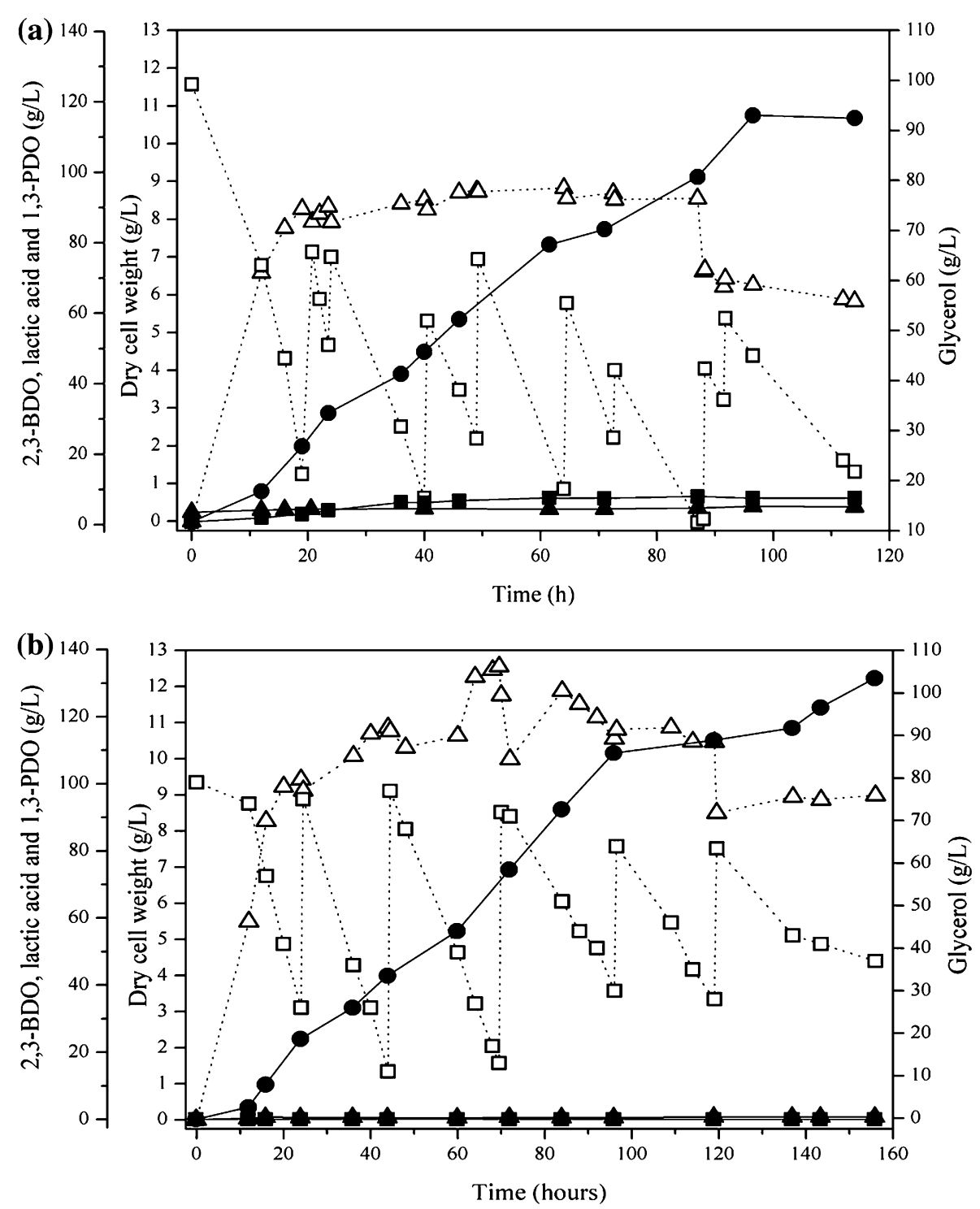

Fig. 4 Time course of 2,3-BDO fermentation using pure glycerol and crude glycerol by K. oxytoca M1 $\Delta p d u C \Delta / d h A$. a Fed-batch fermentation with pure glycerol. b Fed-batch fermentation with crude glycerol. The following symbols were used: dry cell weight unfilled triangle, residual glycerol unfilled square, 2,3-BDO filled circle, 1,3-PDO filled square and lactic acid filled triangle

by the deletion of $p d u C$ and $l d h A$, probably because increased NADH availability would induce the NADHconsuming ethanol synthesis pathway for NADH/NAD ${ }^{+}$ balance. The production of 1,3-PDO (6.9 g/L) by $K . o x y-$ toca M3 was lower than that of $K$. oxytoca M1 $(8.9 \mathrm{~g} / \mathrm{L})$, but it was higher than that of $K$. oxytoca M2 $(0.8 \mathrm{~g} / \mathrm{L})$ (Fig. 3). This result suggests that an alternative glycerol dehydratase other than PduC might be involved in the production of 1,3-PDO to maintain $\mathrm{NADH} / \mathrm{NAD}^{+}$balance in $K$. oxytoca M3. However, considering that the concentration ratio of 1,3-PDO to 2,3-BDO obtained with $K$. oxytoca M3 was much lower than that obtained with $K$. oxytoca M1 (0.06:1 vs. $0.15: 1$ in 1,3-PDO:2,3$\mathrm{BDO}$ ratio), the deletion of $p d u C$ was still effective to decrease carbon flux to 1,3-PDO.

It has been known that a mixture of meso-2,3-BDO (consuming $1 \mathrm{~mol}$ of NADH per mole of 2,3-BDO production) and (2S,3S)-2,3-BDO (consuming $2 \mathrm{~mol}$ of $\mathrm{NADH}$ per mole of 2,3-BDO production) is produced by K. oxytoca [2] (Fig. 1). Because the excess NADH due to the significantly deceased NADH-requiring metabolite production (lactic acid and 1,3-PDO) should be consumed to maintain the redox balance, we analyzed the ratio of (2S,3S)-2,3-BDO to meso-2,3-BDO. Interestingly, 
the ratio of $(2 \mathrm{~S}, 3 \mathrm{~S})-2,3-\mathrm{BDO}$ to meso-2,3-BDO for $K$. oxytoca M3 was higher than $K$. oxytoca M1 (1.04:1 vs. $0.79: 1$ ), implying that $K$. oxytoca M3 consumed more $\mathrm{NADH}$ than $K$. oxytoca M1 per mole of 2,3-BDO production (1.51 vs. $1.44 \mathrm{~mol}$ of $\mathrm{NADH}$ ). In fact, assuming that the aerobic route was involved to convert glycerol to pyruvate (i.e., $1 \mathrm{~mol}$ of $\mathrm{NADH}$ generation per mole of glycerol) (Fig. 1), the NADH recovery based on the total consumed NADH for metabolite production (2,3-BDO, 1,3-PDO, lactic acid, and ethanol) and total NADH generation from glycerol were similar for $K$. oxytoca M3 and K. oxytoca M1 (77 vs. 75 \%). However, the percentage of NADH consumption toward 2,3-BDO production out of total NADH consumption in K. oxytoca M3 was much higher than in K. oxytoca M1 (accounting for 79 and $65 \%$ of total NADH consumption, respectively). This higher NADH consumption due to the higher (2S,3S)-2,3-BDO portion by $K$. oxytoca M3 would explain how the redox balance was maintained despite the deletion of $p d u C$ and ldhA.

In the meantime, we found that the ratio of $(2 \mathrm{~S}, 3 \mathrm{~S})-2,3-$ BDO to meso-2,3-BDO produced by $K$. oxytoca M1 was 0.11:1 and 1.06:1 from glucose and glycerol, respectively, in flask cultures (Additional file 1: Fig. S3). When K. oxytoca KCTC1686 (99.5 \% 16S rDNA sequence similarity to K. oxytoca M1) [26] was tested, the ratio of (2S,3S)-2,3BDO to meso-2,3-BDO produced was $0.11: 1$ and $0.44: 1$ from glucose and glycerol, respectively. Therefore, the high production of 2,3-BDO by $K$. oxytoca $\mathrm{M} 1$ and $K$. oxytoca M3 from glycerol compared to other Klebsiella strains might be partially attributed to the regulation of redox balance by significantly increasing the (2S,3S)-2,3BDO portion.

\section{High production of 2,3-BDO using crude glycerol by $K$. oxytoca M3 in fed-batch fermentation}

As seen in Fig. 2, crude glycerol was more effective in 2,3BDO production by $K$. oxytoca M1. Therefore, fed-batch fermentation using crude glycerol was carried out with $K$. oxytoca M3 to investigate whether a higher 2,3-BDO production would be achieved in comparison to the results in Fig. 4a.

Unlike 2,3-BDO production from pure glycerol by $K$. oxytoca M3, which stopped after $114 \mathrm{~h}, 2,3-\mathrm{BDO}$ production from crude glycerol occurred until $156 \mathrm{~h}$. When crude glycerol was used as the sole carbon source for $K$. oxytoca M3, DCW was much higher than those obtained with pure glycerol (Table 2). Furthermore, 2,3-BDO concentration $(131.5 \mathrm{~g} / \mathrm{L})$ and yield $(0.44 \mathrm{~g} / \mathrm{g})$ were higher than those obtained with pure glycerol by 1.13 -fold and 1.14-fold, respectively. The 2,3-BDO productivity obtained using crude glycerol was similar to that obtained with pure glycerol during about $100 \mathrm{~h}$ of fermentation
(1.10-1.15 g/L/h), but decreased to $0.84 \mathrm{~g} / \mathrm{L} / \mathrm{h}$ as the fermentation was prolonged up to $156 \mathrm{~h}$.

As expected, only a small amount of lactic acid $(0.8 \mathrm{~g} / \mathrm{L})$ was produced. Notably, in contrast to the fed-batch fermentation using pure glycerol by K. oxytoca M3 (Fig. 4a), 1,3-PDO was not detected during $156 \mathrm{~h}$ of fed-batch fermentation using crude glycerol (Fig. 4b). Moreover, ethanol production $(1.9 \mathrm{~g} / \mathrm{L})$ from crude glycerol was much lower than that from pure glycerol $(9.3 \mathrm{~g} / \mathrm{L})$ (Table 2$)$. This decrease of net NADH-consuming by-product formation (1,3-PDO and ethanol, Additional file 1: Table S1) under aerobic condition was not likely attributed to the increase of NADH consumption toward 2,3-BDO production, because the ratio of $(2 \mathrm{~S}, 3 \mathrm{~S})-2,3-\mathrm{BDO}$ to meso2,3-BDO (0.92:1) using crude glycerol was slightly lower than that using pure glycerol (1.04:1). It is not clear why crude glycerol is more effective than pure glycerol in 2,3BDO production with less formation of by-product. A possible reason would be the presence of nutrients stimulating cell growth. Considering the enhanced cell growth with crude glycerol, the regeneration of $\mathrm{NAD}^{+}$via electron transport chain under aerobic conditions might be increased to generate more energy (i.e., ATP). Because of this additional NADH consumption, the production of net NADH-consuming by-product might be decreased. Further investigation would be needed to elucidate the effect of crude glycerol on metabolism.

Table 4 compares the 2,3-BDO production from glycerol as the sole carbon source or the mixture of glycerol and sugar provided as a co-substrate from previous reports and this study. Until now, Petrov and Petrova [21] reported the highest production of 2,3-BDO $(70 \mathrm{~g} / \mathrm{L})$ by $K$. pneumoniae G31 using pure glycerol as the sole carbon source with a yield of $0.39 \mathrm{~g} / \mathrm{g}$ and productivity of $0.47 \mathrm{~g} / \mathrm{L} / \mathrm{h}$ through the application of forced $\mathrm{pH}$ fluctuation. Yang et al. [25] obtained a high concentration $(83.3 \mathrm{~g} / \mathrm{L})$, yield $(0.42 \mathrm{~g} / \mathrm{g})$ and productivity $(0.87 \mathrm{~g} / \mathrm{l} / \mathrm{h})$ of 2,3-BDO by $B$. amyloliquefaciens using beet molasses as a co-substrate in addition to biodiesel-derived glycerol; however, using crude glycerol as the sole carbon source, B. amyloliquefaciens produced only $43.1 \mathrm{~g} / \mathrm{L}$ of $2,3-\mathrm{BDO}$ with a yield of $0.38 \mathrm{~g} / \mathrm{g}$ and productivity of $0.45 \mathrm{~g} / \mathrm{L} / \mathrm{h}$. In this study, the efficient 2,3-BDO production from biodiesel-derived glycerol was fulfilled by the deletion of the $p d u C$ and $l d h A$ genes in K. oxytoca M3 to reduce the formation of 1,3-PDO and lactic acid, and consequently the carbon flux was mainly redirected to 2,3-BDO. More importantly, use of the double deletion mutant and crude glycerol resulted in 1,3-PDO-free 2,3-BDO production, involving no concern to separate 1,3-PDO from 2,3-BDO-containing cell broth.

Overall, a new record of the highest 2,3-BDO concentration from crude glycerol $(131.5 \mathrm{~g} / \mathrm{L})$ as the sole 
Table 4 Comparison of 2,3-BDO production from glycerol

\begin{tabular}{|c|c|c|c|c|c|c|c|}
\hline \multirow[t]{2}{*}{ Host bacteria } & \multirow[t]{2}{*}{ Carbon sources } & \multirow[t]{2}{*}{ Culture mode } & \multicolumn{2}{|c|}{ Concentration (g/L) } & \multirow{2}{*}{$\begin{array}{l}\text { 2,3-BDO productivity } \\
\text { (g/LH) }\end{array}$} & \multirow{2}{*}{$\begin{array}{l}\text { 2,3-BDO } \\
\text { yield }(g / g)\end{array}$} & \multirow[t]{2}{*}{ References } \\
\hline & & & 2,3-BDO & 1,3-PDO & & & \\
\hline K. pneumoniae & Pure glycerol & Fed batch & 49.2 & 10.6 & 0.18 & 0.36 & {$[20]$} \\
\hline K.pneumoniae & Pure glycerol & Fed batch & 70 & 16.3 & 0.47 & 0.39 & {$[21]$} \\
\hline B. amyloliquefaciens & Crude glycerol & Fed batch & 43.1 & - & 0.45 & 0.38 & {$[25]$} \\
\hline B. amyloliquefaciens & Crude glycerol + molasses & Fed batch & 83.3 & - & 0.87 & 0.42 & {$[25]$} \\
\hline K. oxytoca & Crude glycerol & Batch flask & 4.8 & 8.4 & & 0.14 & {$[19]$} \\
\hline K. oxytoca & Crude glycerol + glucose & Batch flask & 8.0 & 3.8 & & 0.22 & {$[19]$} \\
\hline K. oxytoca M3 & Pure glycerol & Fed batch & 115.0 & 6.9 & 1.01 & 0.39 & This study \\
\hline K. oxytoca M3 & Crude glycerol & Fed batch & 131.5 & 0 & 0.84 & 0.44 & This study \\
\hline
\end{tabular}

carbon source was achieved with the highest productivity $(0.84 \mathrm{~g} / \mathrm{L} / \mathrm{h})$ and yield $(0.44 \mathrm{~g} / \mathrm{g}$ crude glycerol) without $1,3-\mathrm{PDO}$ production. It is worth noting that $2,3-\mathrm{BDO}$ concentration obtained using crude glycerol in this study is comparable to the 2,3-BDO production from glucose reported by Ji et al. (130 g/L) and Park et al. (113 g/L) using $K$. oxytoca strains $[6,8]$. Further improvement in 2,3-BDO productivity would make it more feasible to produce 2,3-BDO from biodiesel-derived glycerol for industrial use.

\section{Conclusions}

In this study, biodiesel-derived glycerol was used as the sole carbon source for 2,3-BDO production by the engineered strain $K$. oxytoca M3. Enhanced 2,3-BDO production from crude glycerol was achieved by disruption of the $p d u C$ and $l d h A$ genes, which resulted in a nearly abolished lactic acid and 1,3-PDO production. To the best of our knowledge, 2,3-BDO concentration (131.5 g/L), productivity $(0.84 \mathrm{~g} / \mathrm{L} / \mathrm{h})$, and yield $(0.44 \mathrm{~g} / \mathrm{g})$ achieved in this study are the highest levels in glycerol-based 2,3$\mathrm{BDO}$ production reported to date, demonstrating that biodiesel-derived glycerol could be used to produce 2,3BDO cost-effectively by the metabolically engineered strain K. oxytoca M3.

\section{Methods}

\section{Microorganisms and media}

All bacterial strains and plasmids used in this study are listed in Table 3. K. oxytoca M1 was deposited in the Korean Culture Center of Microorganisms (KCCM) as KCCM 1177P. K. oxytoca KCTC1686 (equivalent to ATCC8724) was purchased from the Korean

Collection for type culture (KCTC, Korea). K. oxytoca M1 and its mutants were pre-cultured in Luria-Bertani (LB) medium at $30^{\circ} \mathrm{C}$. The defined medium used for flask fermentation contained (per L of distilled water): $\mathrm{K}_{2} \mathrm{HPO}_{4}$ $13.7 \mathrm{~g}, \mathrm{KH}_{2} \mathrm{PO}_{4} 2 \mathrm{~g},\left(\mathrm{NH}_{4}\right)_{2} \mathrm{HPO}_{4} 3.3 \mathrm{~g},\left(\mathrm{NH}_{4}\right)_{2} \mathrm{SO}_{4} 6.6 \mathrm{~g}$,
$\mathrm{MgSO}_{4} \cdot 7 \mathrm{H}_{2} \mathrm{O} 0.25 \mathrm{~g}, \mathrm{FeSO}_{4} \cdot 7 \mathrm{H}_{2} \mathrm{O} 0.05 \mathrm{~g}, \mathrm{ZnSO}_{4} \cdot 7 \mathrm{H}_{2} \mathrm{O}$ $0.001 \mathrm{~g}, \mathrm{MnSO}_{4} \cdot \mathrm{H}_{2} \mathrm{O} 0.01 \mathrm{~g}, \mathrm{CaCl}_{2} \cdot 2 \mathrm{H}_{2} \mathrm{O} 0.01 \mathrm{~g}$, and EDTA 0.05 g. Pure glycerol or crude glycerol were added to the defined medium as needed. Crude glycerol provided by GS Caltex Corporation (South Korea) contained (wt/wt): $81.7 \%$ of glycerol, $10.5 \%$ of water, $5 \%$ of MONG (matter organic non-glycerol), $2.9 \%$ of ash, $2.4 \%$ of sodium, and less than $0.01 \%$ of methanol, magnesium and potassium. The medium used for $\mathrm{pH}$-controlled fermentation was the defined medium supplemented with $5 \mathrm{~g} / \mathrm{L}$ yeast extract and $10 \mathrm{~g} / \mathrm{L}$ casamino acid [16].

\section{Fermentation procedures (flask and fermentor)}

For flask fermentation without $\mathrm{pH}$ control, pre-culture cultivation was carried out overnight in LB medium at $30{ }^{\circ} \mathrm{C}$ and $200 \mathrm{rpm}$. Then, the seed culture was inoculated in a $100 \mathrm{~mL}$ Erlenmeyer flask (5 \%, v/v) containing $20 \mathrm{~mL}$ of the defined medium. Pure glycerol and crude glycerol (35-40 g/L) were added to the defined medium to investigate metabolite production patterns (initial $\mathrm{pH}$ $7.0,30{ }^{\circ} \mathrm{C}, 200 \mathrm{rpm}, 48 \mathrm{~h}$ ). All flask experiments were performed in triplicate.

All trials for 2,3-BDO fermentation with $\mathrm{pH}$ control in this study were conducted in a $3 \mathrm{~L}$ stirred fermenter (Fermentec FMT ST, South Korea) with a working volume of $1 \mathrm{~L}$. For seed culture preparation, K. oxytoca M1 and the derivatives were inoculated into $100 \mathrm{~mL}$ of $\mathrm{LB}$ medium and cultivated on a rotary shaker at $200 \mathrm{rpm}$ at $30{ }^{\circ} \mathrm{C}$ for $10 \mathrm{~h}$. The seed culture $(10 \%$, v/v) was then inoculated into the defined medium supplemented with $10 \mathrm{~g} / \mathrm{L}$ casamino acid and $5 \mathrm{~g} / \mathrm{L}$ yeast extract. All cultivations were carried out at $30{ }^{\circ} \mathrm{C}$, and the $\mathrm{pH}$ level was maintained at 6 by automatic addition of $5 \mathrm{~N} \mathrm{NaOH}$. The aeration rate was controlled at 1.0 volume of air per volume of liquid per minute ( $\mathrm{vvm}$ ) with the agitation speed of $400 \mathrm{rpm}$.

The batch cultivation was carried out at $30{ }^{\circ} \mathrm{C}$ using fermentation medium containing $90 \mathrm{~g} / \mathrm{L}$ of glycerol. 
Fed-batch fermentation was carried out with an initial glycerol concentration of $90-100 \mathrm{~g} / \mathrm{L}$, and then a concentrated solution containing $800 \mathrm{~g} / \mathrm{L}$ of pure glycerol or crude glycerol was fed into the fermenter as required.

\section{Construction of the pduC deletion mutant}

The $p d u C$ gene (accession number AIE72369) deletion mutant, $K$. oxytoca M2, was developed from $K$. oxytoca M1 using the $\lambda$ Red recombination method [30, 31] (Additional file 1: Fig. S1A). The full genome sequence of K. oxytoca M1 (CP008841) was provided by Macrogen Inc. (Seoul, South Korea) [32]. Two PCR products including the upstream and downstream regions of $p d u C$ in genomic DNA were generated using the primers pduUp1 and pduUp2 to get $1031 \mathrm{bp}$ of fragments and using the primers pduDown 1 and pduDown 2 to obtain $843 \mathrm{bp}$ of fragments, respectively (Table 5). In addition, PCR using the pTOP-FCF plasmid as a template was performed with the primers pduCUDFCF1 and pduCUDFCF2 (Table 5) to get $930 \mathrm{bp}$ of the PCR products (step 1 in Additional file 1: Fig. S1A). The $2639 \mathrm{bp}$ fragments spanning the upstream regions (1031 bp), FRT-Cm ${ }^{\mathrm{R}}$-FRT (930 bp), and downstream regions ( $843 \mathrm{bp}$ ) were amplified by the

Table 5 Oligonucleotides used in this study

\begin{tabular}{|c|c|c|}
\hline Oligonucleotides & Sequence $^{a}$ & Source \\
\hline pduCUp1 & 5'-TTATGCTTCTTTTTTACGCAGCTTATCG-3 & 'This study \\
\hline pduCUp2 & $\begin{array}{l}\text { 5'-TTTCTAGAGAATAGGAACTTC }^{\text {GGGGAACTGCATCATGGAA-3' }}\end{array}$ & This study \\
\hline pduCUDFCF1 & $\begin{array}{l}\text { 5'-TTCCATGATGCAGTTCCCC } \\
\text { GAAGTTCCTATTCTCTAGAAA-3' }\end{array}$ & This study \\
\hline pduCUDFCF2 & $\begin{array}{l}\text { 5'-GGCACAATTTTTTAATCTTA } \\
\text { GAAGTTCCTATACTTTCTAGA-3' }\end{array}$ & This study \\
\hline pduCDown1 & $\begin{array}{l}5^{\prime} \text {-TCTAGAAAGTATAGGAACTTC } \\
\text { TAAGATTAAAAAATTGTGCC-3' }\end{array}$ & This study \\
\hline pduCDown2 & $\begin{array}{l}\text { 5'-AATAAGCCTCAGAAAATTGAGTTA } \\
\text { GAAATAAAGTTGA-3' }\end{array}$ & This study \\
\hline pduCcon1 & 5'-CCGTTTTCAACCAGCGTCAG-3' & This study \\
\hline pduCcon2 & 5'-CCGGTAATTCTCACCCGGAG-3' & This study \\
\hline IdhADown1 & 5'-TTACCAGACCACGGATTGCG-3' & This study \\
\hline IdhADown2 & $\begin{array}{l}5^{\prime} \text {-СTTTCTAGAGAATAGGAACTTC } \\
\text { CTTTCCCTTTTGTGCTCCT-3' }\end{array}$ & This study \\
\hline IdhAUDFCF1 & $\begin{array}{l}\text { 5'-GGAGCACAAAAGGGAAAG } \\
\text { GAAGTTCCTATTCTCTAGAAAG-3' }\end{array}$ & This study \\
\hline IdhAUDFCF2 & $\begin{array}{l}\text { 5'-TATTATCACTGGAGAAAAGTCTT } \\
\text { GAAGTTCCTATACTTTCTAGAGAA-3' }\end{array}$ & This study \\
\hline IdhAUP1 & $\begin{array}{l}\text { 5'-TTCTCTAGAAAGTATAGGAACTTC } \\
\text { AAGACTTTTCTCCAGTGATAATA-3' }\end{array}$ & This study \\
\hline IdhAUP2 & 5'-GCGGGCTTTCATTGAGTGAG-3' & This study \\
\hline IdhAcon 1 & 5'-GGGAATTGTAACTTTATCGCAGGC-3' & This study \\
\hline IdhAcon2 & 5'-CCGTGACGGTATTATCACTGGA-3' & This study \\
\hline
\end{tabular}

a Underlined sequences are homologous with the FRT region primers pduCDown 2 and pduCUP1, using PCR products as a template by the overlap extension PCR method.

Then, the $\lambda$ Red recombinase expression plasmid pRedET was transformed into $K$. oxytoca M1 by electroporation at $12.5 \mathrm{kV} / \mathrm{cm}, 200 \Omega, 25 \mu \mathrm{F}$ (Gene PulserXcell, Biorad). The 2639 bp linear fragments containing $\mathrm{Cm}^{\mathrm{R}}$ were transformed to K. oxytoca M1 haboring pRedET, and the chloramphenicol-resistant colonies were selected on LB agar plates containing $25 \mu \mathrm{g} / \mathrm{mL}$ of chloramphenicol at $37{ }^{\circ} \mathrm{C}$ (step 2 and step 3 in Additional file 1: Fig. $\mathrm{S} 1 \mathrm{~A})$. Deletion of the $p d u C$ gene from the chromosome was confirmed by the size of the PCR product (1010 bp) obtained using the pduCcon1 and pduCcon2 primer pair (step 3 in Additional file 1: Fig. S1A, Table 5). To remove the $\mathrm{Cm}^{\mathrm{R}}$ cassette from the chromosome, FLP expression plasmids (707-FLPe, Gene Brideges, Germany) were transformed into the cells and the tetracycline-resistant transformants were selected at $30{ }^{\circ} \mathrm{C}$. After cultivation at $42{ }^{\circ} \mathrm{C}$ overnight, the desirable antibiotic-susceptible transformants which lost the FRT flanking $\mathrm{Cm}^{\mathrm{R}}$ gene and the 707-FLPe were selected. The resulting mutant was confirmed by PCR (153 bp, step 4 in Additional file 1: Fig. S1A) using the pduCcon 1 and pduCcon 2 primer pair (Table 5).

\section{The $p d u C$ and IdhA double deletion mutant}

The $p d u C$ and $l d h A$ (encoding lactate dehydrogenase, accession number AIE70186) double deletion mutant, $K$. oxytoca M3, was constructed from $K$. oxytoca M2 ( $K$. oxytoca $\mathrm{M} \Delta p d u C$ ) using the $\lambda$ Red recombination method as described above. Two PCR products including the upstream and downstream regions of ldhA in genomic DNA were generated by using the primers ldhAUp1 and ldhAUp2 (Table 5) to get $993 \mathrm{bp}$ of PCR products and by using the primers ldhADown1 and ldhADown2 (Table 5) to obtain 1091 bp of PCR products (step 1 in Additional file 1: Fig. S1B), respectively. In addition, the $932 \mathrm{bp}$ of PCR products were generated by using primers (ldhAUDFCF1 and ldhAUDFCF2) that included homology extensions to the upstream and downstream regions and priming sequences for pTOPFCF as a template (Table 5). The $2665 \mathrm{bp}$ fragments were amplified by the primer pair, ldhADown1 and ldhAUP2, using PCR products (993, 932, and $1091 \mathrm{bp}$ ) as a template by the overlap extension PCR method.

After construction of linear fragments for homologous recombination of the $l d h A$ gene, the next process was conducted in the same manner as the $p d u C$ deletion mutant construction process (step 2 and 3 in Additional file 1: Fig. S1B). Deletion of the $l d h A$ gene from the chromosome was confirmed by PCR (150 bp, step 4 in Additional file 1: Fig. S1B) using the ldhAcon1 and ldhAcon2 primer pair (Table 5). 


\section{Analytical methods}

Dry cell weight (DCW, $g / \mathrm{L}$ ) was calculated from optical density at $600 \mathrm{~nm}\left(\mathrm{OD}_{600}\right)$ using the calibration curve of $\mathrm{OD}_{600}$ and the dry cell weight of $K$. oxytoca M1. The $\mathrm{OD}_{600}$ of the broth was measured by UV-visible spectrophotometry (Cary $60 \mathrm{UV}-\mathrm{Vis}$, Agilent Technologies, USA) with appropriate dilution.

The concentrations of meso-2,3-BDO, (2S,3S)-2,3-BDO, (2R,3R)-2,3-BDO, 1,3-PDO, and ethanol were measured using a gas chromatograph (Shimadzu GC-2010, Kyoto, Japan) equipped with a flame ionized detector (FID) and an HP-Chiral-20B GC column (30 m X $0.32 \mathrm{~mm} \mathrm{X}$ $0.25 \mu \mathrm{m}$ ) (Agilent) under the conditions described previously $[26,27]$. Glycerol and lactic acid were analyzed using a high-performance liquid chromatograph (HPLC) (Agilent 1260, Waldbronn, Germany) equipped with a refractive index detector (RID) and an Aminex HPX-87 H Ion Exclusion Column $(300 \times 7.8 \mathrm{~mm}$, Bio-Rad, Hercules, CA, USA) under the following conditions: sample volume of $20 \mu \mathrm{L}$, mobile phase of $5 \mathrm{mM} \mathrm{H}_{2} \mathrm{SO}_{4}$, flow rate of $0.5 \mathrm{~mL} / \mathrm{min}$, and column temperature of $50^{\circ} \mathrm{C}$. All culture samples tested were preliminarily filtered through a membrane filter $(0.45 \mu \mathrm{m}$ pore size, Millipore, USA).

\section{Additional file}

Additional file 1: Figure S1. Schematic representation for generating of deletion mutants using the $\lambda$ Red recombination method. Figure S2. PCR verification for the deletion of $p d u C$ and IdhA genes from $K$. oxytoca $M 1$ and $K$. oxytoca $M 2$, respectively. Figure S3. Relative production of meso2,3-BDO and (2S, 3S)-2,3-BDO by K. oxytoca M1 and K. oxytoca KCTC1686 using glucose or glycerol as the carbon sources. Table S1. Net NADH balance for the corresponding product formation per mole of glycerol.

\section{Abbreviations}

2,3-BDO: 2,3-butanediol; 1,3-PDO: 1,3-propanediol; PduC: glycerol dehydratase large subunit; LDH: lactate dehydrogenase; DCW: dry cell weight; bp: base pair; PCR: polymerase chain reaction; NADH: nicotinamide adenine dinucleotide plus hydrogen; $\mathrm{OD}_{600}$ : Optical density at $600 \mathrm{~nm}$.

\section{Authors' contributions}

SC, HW, JL, and YU conceived the study. SC carried out the experimental works and coordinated the manuscript draft. TK participated in the experimental work. YK helped in the analysis. HW, JL, YK, and YU reviewed and commented on the manuscript. YU participated in its design and coordination of the manuscript draft. All authors read and approved the final manuscript.

\footnotetext{
Author details

${ }^{1}$ Clean Energy Research Center, Korea Institute of Science and Technology (KIST), Hwarangno 14-gil 5, Seongbuk-gu, Seoul 136-791, Republic of Korea. ${ }^{2}$ Interdisciplinary Program in Agriculture Biotechnology, Collage of Agriculture and Life Science, Seoul National University, 1 Gwanak-ro, Gwanak-gu, Seoul, Republic of Korea. ${ }^{3}$ Clean Energy and Chemical Engineering, Korea University of Science and Technology, 217 Gajeong-ro, Yuseong-gu, Daejeon, Republic of Korea. ${ }^{4}$ Department of Chemical and Biomolecular Engineering, Sogang University, 35 Baekbeom-ro, Mapo-gu, Seoul, Republic of Korea.
}

\section{Acknowledgements}

This work was supported by the R\&D program of MKE/KEIT (no. 10035578, Development of 2,3-butanediol and derivative production technology for the
C-zero bio-platform industry) and the KIST Institutional Program (Project No. 2E25560).

\section{Compliance with ethical guidelines}

\section{Competing interests}

The authors declare that they have no competing interests.

Received: 8 June 2015 Accepted: 4 September 2015

Published online: 15 September 2015

\section{References}

1. Hermann BG, Patel M. Today's and tomorrow's bio-based bulk chemicals from white biotechnology. Appl Biochem Biotechnol. 2007;136:361-88.

2. Ji XJ, Huang H, Ouyang PK. Microbial 2,3-butanediol production: a stateof-the-art review. Biotechnol Adv. 2011;29:351-64.

3. Xiu Z-L, Zeng A-P. Present state and perspective of downstream processing of biologically produced 1, 3-propanediol and 2, 3-butanediol. Appl Microbiol Biotechnol. 2008;78:917-26.

4. Haveren JV, Scott EL, Sanders J. Bulk chemicals from biomass. Biofuels Bioprod Biorefin. 2008;2:41-57.

5. Ma C, Wang A, Qin J, Li L, Ai X, Jiang T, et al. Enhanced 2,3-butanediol production by Klebsiella pneumoniae SDM. Appl Microbiol Biotechnol. 2009:82:49-57.

6. Ji XJ, Huang H, Zhu JG, Ren LJ, Nie ZK, Du J, et al. Engineering Klebsiella oxytoca for efficient 2, 3-butanediol production through insertional inactivation of acetaldehyde dehydrogenase gene. Appl Microbiol Biotechnol. 2010;85:1751-8.

7. Kim B, Lee S, Park J, Lu M, Oh M-K, Kim Y, et al. Enhanced 2,3-Butanediol production in recombinant Klebsiella pneumoniae via overexpression of synthesis-related genes. J Microbiol Biotechnol. 2012;22:1258-63.

8. Park JM, Song H, Lee HJ, Seung D. In silico aided metabolic engineering of Klebsiella oxytoca and fermentation optimization for enhanced 2,3-butanediol production. J Ind Microbiol Biotechnol. 2013;40:1057-66.

9. Guo $X, C a o C$, Wang $Y, L i C$, Wu M, Chen $Y$, et al. Effect of the inactivation of lactate dehydrogenase, ethanol dehydrogenase, and phosphotransacetylase on 2,3-butanediol production in Klebsiella pneumoniae strain. Biotechnol Biofuels. 2014;7:44

10. Li L, Zhang L, Li K, Wang Y, Gao C, Han B, et al. A newly isolated Bacillus licheniformis strain thermophilically produces 2,3-butanediol, a platform and fuel bio-chemical. Biotechnol Biofuels. 2013;6:123.

11. Zhang $L$, Yang $Y$, Ja Sun, Shen $Y$, Wei D, Zhu J, et al. Microbial production of 2,3-butanediol by a mutagenized strain of Serratia marcescens H3O. Bioresour Technol. 2010;101:1961-7.

12. Cheng K-K, Liu Q, Zhang J-A, Li J-P, XU J-M, Wang G-H. Improved 2, 3-butanediol production from corncob acid hydrolysate by fed-batch fermentation using Klebsiella oxytoca. Process Biochem. 2010;45:613-6.

13. Li L, Li K, Wang K, Chen C, Gao C, Ma C, et al. Efficient production of 2 , 3-butanediol from corn stover hydrolysate by using a thermophilic BacilIus licheniformis strain. Bioresour Technol. 2014;170:256-61.

14. Sun L-H, Wang X-D, Dai J-Y, Xiu Z-L. Microbial production of 2, 3-butanediol from Jerusalem artichoke tubers by Klebsiella pneumoniae. Appl Microbiol Biotechnol. 2009;82:847-52.

15. Jiang L-Q, Fang Z, Guo F. Yang L-b. Production of 2, 3-butanediol from acid hydrolysates of Jatropha hulls with Klebsiella oxytoca. Bioresour Technol. 2012;107:405-10.

16. Jung M-Y, Park B-S, Lee J, Oh M-K. Engineered Enterobacter aerogenes for efficient utilization of sugarcane molasses in 2,3-butanediol production. Bioresour Technol. 2013;139:21-7.

17. Ahn J-H, Sang B-I, Um Y. Butanol production from thin stillage using Clostridium pasteurianum. Bioresour Technol. 2011;102:4934-7.

18. Da Silva GP, Mack M, Contiero J. Glycerol: a promising and abundant carbon source for industrial microbiology. Biotechnol Adv. 2009;27:30-9.

19. Metsoviti M, Paraskevaidi K, Koutinas A, Zeng A-P, Papanikolaou S. Production of 1, 3-propanediol, 2, 3-butanediol and ethanol by a newly isolated Klebsiella oxytoca strain growing on biodiesel-derived glycerol based media. Process Biochem. 2012;47:1872-82. 
20. Petrov K, Petrova P. High production of 2,3-butanediol from glycerol by Klebsiella pneumoniae G31. Appl Microbiol Biotechnol. 2009;84:659-65.

21. Petrov K, Petrova P. Enhanced production of 2,3-butanediol from glycerol by forced pH fluctuations. Appl Microbiol Biotechnol. 2010;87:943-9.

22. Jun S-A, Moon C, Kang C-H, Kong SW, Sang B-I, Um Y. Microbial fed-batch production of 1, 3-propanediol using raw glycerol with suspended and immobilized Klebsiella pneumoniae. Appl Biochem Biotechnol. 2010;161:491-501.

23. Ashok S, Sankaranarayanan M, Ko Y, Jae K-E, Ainala SK, Kumar V, et al. Production of 3-hydroxypropionic acid from glycerol by recombinant Klebsiella pneumoniae $\Delta$ dhaT $\Delta y a h D$ which can produce vitamin B12 naturally. Biotechnol Bioeng. 2013;110:511-24.

24. Yen H-W, Li F-T, Chang J-S. The effects of dissolved oxygen level on the distribution of 1,3-propanediol and 2,3-butanediol produced from glycerol by an isolated indigenous Klebsiella sp. Ana-WS5. Bioresour Technol. 2014;153:374-8

25. Yang T-W, Rao Z-M, Zhang X, Xu M-J, Xu Z-H, Yang S-T. Fermentation of biodiesel-derived glycerol by Bacillus amyloliquefaciens: effects of co-substrates on 2,3-butanediol production. Appl Microbiol Biotechnol. 2013;97:7651-8.

26. Cho S, Kim KD, Ahn J-H, Lee J, Kim S-W, Um Y. Selective production of 2, 3-butanediol and acetoin by a newly isolated bacterium Klebsiella oxytoca M1. Appl Biochem Biotechnol. 2013;170:1922-33.

27. Moon C, Ahn J-H, Kim SW, Sang B-I, Um Y. Effect of biodiesel-derived raw glycerol on 1, 3-propanediol production by different microorganisms. Appl Biochem Biotechnol. 2010;161:502-10.
28. Chatzifragkou A, Papanikolaou S. Effect of impurities in biodiesel-derived waste glycerol on the performance and feasibility of biotechnological processes. Appl Microbiol Biotechnol. 2012;95:13-27.

29. Yang G, Tian J, Li J. Fermentation of 1, 3-propanediol by a lactate deficient mutant of Klebsiella oxytoca under microaerobic conditions. Appl Microbiol Biotechnol. 2007;73:1017-24.

30. Datsenko KA, Wanner BL. One-step inactivation of chromosomal genes in Escherichia coli K-12 using PCR products. PNAS. 2000;97:6640-5.

31. Lee S, Kim B, Jeong D, Oh M-K, Um Y, Kim Y-R, et al. Observation of 2,3-butanediol biosynthesis in Lys regulator mutated Klebsiella pneumoniae at gene transcription level. J Biotechnol. 2013;168:520-6.

32. Shin SH, Roh H, Kim J, Cho S, Um Y, Lee J, et al. Complete genome sequence of Klebsiella oxytoca M1, isolated from Manripo area of South Korea. J Biotechnol. 2015;198:1-2.

33. Shin SH, Kim S, Kim JY, Lee S, Um Y, Oh M-K, et al. Complete genome sequence of Klebsiella oxytoca KCTC 1686, used in production of 2 3-butanediol. J Bacteriol. 2012;194:2371-2.

34. Jung SG, Jang JH, Kim AY, Lim MC, Kim B, Lee J, et al. Removal of pathogenic factors from 2,3-butanediol-producing Klebsiella species by inactivating virulence-related wabG gene. Appl Microbiol Biotechnol. 2013;5:1997-2007.

\section{Submit your next manuscript to BioMed Central and take full advantage of:}

- Convenient online submission

- Thorough peer review

- No space constraints or color figure charges

- Immediate publication on acceptance

- Inclusion in PubMed, CAS, Scopus and Google Scholar

- Research which is freely available for redistribution

Submit your manuscript at

www.biomedcentral.com/submit

C Biomed Central 